\title{
MTA or calcium hydroxide treatment for immature permanent teeth?
}

\section{Abstracted from \\ Chala S, Abouqal R, Rida S.}

Apexification of immature teeth with calcium hydroxide or mineral trioxide aggregate: systematic review and meta-analysis. Oral Surg Oral Med Oral Pathol Oral Radiol Endod 2011; 112: e36-42 Jul 20. [Epub ahead of print].

Address for correspondence: Sanaa Chala, Faculté de Medecine Dentaire de Rabat, Rabat Instituts, BP: 6212 Rabat, Morocco. E-mail: dr_chala@yahoo.fr

\section{Question: In permanent teeth with open apex is calcium hydroxide or MTA more effective in inducing apexification?}

Data sources Pubmed, Medline and SCOPUS databases together with hand searching of the identified key journals' indexes, bibliographies, special issues and reference lists of identified articles were scanned to identify other potentially relevant articles.

Study selection Controlled trials comparing calcium hydroxide versus MTA in immature permanent teeth where the aim was apexification where the outcome was evaluated by clinical symptoms and radiographic evidence and the formation of apical barrier was recorded, were included.

Data extraction and synthesis Following data extraction consensus agreement was used to resolve disagreements. The principal measure of treatment effect was risk difference. The overall effect was tested by using $Z$ score. Heterogeneity was tested by using the $X^{2}$ statistic and I square $\left(I^{2}\right)$. A fixed-effect model was used when the studies in the subgroup were sufficiently similar. A random-effects model was used in the summary analysis when there was heterogeneity between the subgroups.

Results Two studies comparing the two materials with a total of 50 teeth met inclusion criteria. There was no significant difference in either the clinical or radiographic success between the two materials.

Conclusions Calcium hydroxide and MTA may be suitable materials for the treatment of the immature teeth. However, more studies evaluating the factors influencing success and failure in teeth should be conducted.

\section{Commentary}

Mineral trioxide aggregate (MTA) was originally formulated as a root-end filling material for periradicular surgery. ${ }^{1}$ The uses of MTA have since expanded to include the management of open apex cases; this was compared with calcium hydroxide $(\mathrm{CH})$ in this article. The authors enumerated the challenges of managing non-vital immature teeth but since these are not deciduous teeth, the word 'permanent' should have been included, especially in the title.

To prevent overfilling during root canal treatment, $\mathrm{CH}$ is used for apexification, to induce an apical hard tissue barrier. However, MTA is used as an apical plug technique, to obviate the need to achieve apical barrier formation. The authors alluded to the subtle differ- ences but the article's purpose was still stated as '...to compare the efficacy of calcium hydroxide and MTA for root-end induction....'

Only two studies met the inclusion criteria; the numbers treated are small and the follow-up relatively short. With El-Meligy \& Avery, it is arguable that it is necessary to detect radiographically a barrier with MTA because if the periradicular lesion subsequently heals, this is indicative of a favourable outcome. With Pradhan et al., it is arguable that the lack of radiographic evidence of a hard tissue barrier should be considered a failure. Cases were assessed clinically and radiographically every four weeks; this is too soon and too frequent, especially in children.

In the discussion section, a valuable opportunity was missed to provide better coverage on the advantages of MTA over $\mathrm{CH}$ for managing open apex cases: ${ }^{2}$

- reduced number of treatment appointments

- less demanding on both clinical and patient time

- less stressful for the patient in terms of overall treatment duration

- reduced risk of root fracture

- reduced risk of re-infection due to loss of temporary filling.

A possible disadvantage is the greater cost of MTA, but it is more than offset by reduced treatment time.

In the reference list, the same paper was repeated three times (No. $6,25 \& 26$ ). For a systematic review and meta-analysis article, it is unfortunate that a similar Cochrane review ${ }^{3}$ was not mentioned.

Even if barrier techniques continue to be practised, a recent review paper has concluded that MTA appears to overcome the shortcomings of, and may replace, $\mathrm{CH}^{4}{ }^{4}$ However, the advent of regenerative endodontic approaches has already encouraged a paradigm shift in the management of non-vital immature permanent teeth, heralding the beginning of the end of apexification. ${ }^{5}$

Bun San Chong

Institute of Dentistry, Barts \& The London School of Medicine and Dentistry, Queen Mary, University of London, UK

1. Torabinejad M, Hong CU, McDonald F, Pitt Ford TR. Physical and chemical properties of a new root-end filling material. J Endod 1995; 21: 349-353.

2. Pitt Ford HE. Endodontic aspects of traumatic injuries. In Chong BS (ed) Harty's Endodontics in Clinical Practice. $6^{\text {th }}$ ed. pp 209-229. Edinburgh: Churchill Livingstone, Elsevier 2010.

3. Al Ansary MA, Day PF, Duggal MS, Brunton PA. Interventions for treating traumatised non-vital immature permanent front teeth: inducing a calcific barrier and root strengthening (Protocol). Cochrane Database Syst Rev 2005 Issue 4: CD005513.

4. Bakland LK, Andreasen JO. Will mineral trioxide aggregate replace calcium hydroxide in treating pulpal and periodontal healing complications subsequent to dental trauma? A review. Dent Traumatol 2012; 28: 25-32.

5. Huang GT. Apexification: the beginning of its end. Int Endod 2009; 42: 855-866.

Evidence-Based Dentistry (2012) 13, 11. doi:10.1038/sj.ebd.6400838 